Note

\title{
Detritylation of mono- and di-saccharide derivatives using ferric chloride hydrate
}

\author{
Xianglan Ding, Wei Wang, Fanzuo Kong * \\ Research Center for Eco-Environmental Sciences, Academia Sinica, P.O. Box 2871, Beijing 100085, \\ People's Republic of China
}

Received 28 April 1997; accepted 6 June 1997

\begin{abstract}
Quantitative detritylation of mono- and di-saccharide derivatives of diverse structure was achieved with $\mathrm{FeCl}_{3} \cdot 6 \mathrm{H}_{2} \mathrm{O}$ in $\mathrm{CH}_{2} \mathrm{Cl}_{2}$ at room temperature. Benzyl, isopropylidene, isopropylthio, allyl, acetyl, and benzoyl O-protecting groups were not effected under the designated conditions. (C) 1997 Elsevier Science Ltd.
\end{abstract}

Keywords: Detritylation; Ferric chloride hydrate; Iron(III) chloride hydrate

The trityl group has frequently been used for the temporary protection of primary hydroxyl groups due to the generally good crystallizing properties imparted by the trityl ether and its easy removal through mild acid treatment or by hydrogenolysis [1]. However, a great number of articles in the literature indicate that detritylation seems to be a problematic operation. Low yields, formation of byproducts, glycoside cleavage, and especially acyl migration often arise from acid-catalyzed detritylation (e.g., $80 \%$ acetic acid at reflux [2], 80\% formic acid in ethyl acetate at room temperature $[3,4]$, hydrogen chloride in methanol [5] or other solvent [6], and hydrogen hromide in acetic acid [7], among others [8]). In order to avoid hydrolysis or migration, aqueous trifluoroacetic acid [9], sometimes diluted with butanol [10], copper(II) sulfate in boiling benzene [11], powdered anhydrous zinc bromide in dichloromethane [12],

\footnotetext{
${ }^{*}$ Corresponding author
}

boron trifluoride etherate and methanol in aprotic solvents [13], aqueous tetrafluoroboric acid in acetonitrile [14], iodotrimethylsilane [15,16], dimethylaluminum chloride [17] and pyridinium perchlorate in nitromethane-methanol mixtures [18] have all been used as detritylation reagents. Pyridinium perchlorate has been determined to be an effective catalyst for detritylation of acetylated glycosides.

In our recent studies, we were gratified to find the unexpected result that $\mathrm{FeCl}_{3} \cdot 6 \mathrm{H}_{2} \mathrm{O}$ was a highly efficient reagent for detritylation. Compounds of diverse structure, including monopyranoses, furanoses, and disaccharides were tried, and the experiments indicated that benzyl, isopropylidene, isopropylthio, allyl, acetyl, and benzoyl O-protecting groups were quite stable under the designated conditions. No problems with acyl migration, anomerization, or glycosidic bond breaking were observed. Notably, a recent report indicated that anhydrous ferric chloride in dichloromethane under extremely dry conditions can be used for debenzylation in good yield [19]. Also, 
Ikemoto et al. reported anomerization of glycosides with anhydrous $\mathrm{FeCl}_{3}$ [20]. It seems that hydration of the ferric chloride and the use of a relatively small quantity ( 2 equiv in our hands vs. $>4$ equiv in ref. [20]) would account for these differences. Extension of the reaction time to 3 days only caused a little acyl migration, indicating the mildness of the reagent. For the examples cited in this report, most of the starting materials and the products are known, and their identification was easily achieved from their ${ }^{1} \mathrm{H}$ NMR spectra. The starting materials showed trityl phenyl proton signals, while the products showed an upfield shift of H-6 and H-6', and disappearance of the trityl signals. Although most of the compounds involved in the detritylation are known, only limited physicochemical data have been reported in the literature. Here we report the optical rotations of $\mathbf{1 a}, \mathbf{b}-\mathbf{1 1 a}, \mathbf{b}$, together with some data that has appeared in the litcrature (Table 1), and the ${ }^{1}$ H NMR data for most of the compounds. Compounds $\mathbf{3 a}, \mathbf{b}, \mathbf{5 a}, \mathbf{b}, \mathbf{7 a}, \mathbf{b}$, and $\mathbf{1 2} \mathbf{a}, \mathbf{b}$ are intermediates involved in our ongoing synthesis of a natural oligosaccharide, the complete physicochemical data for which will be published elsewhere.
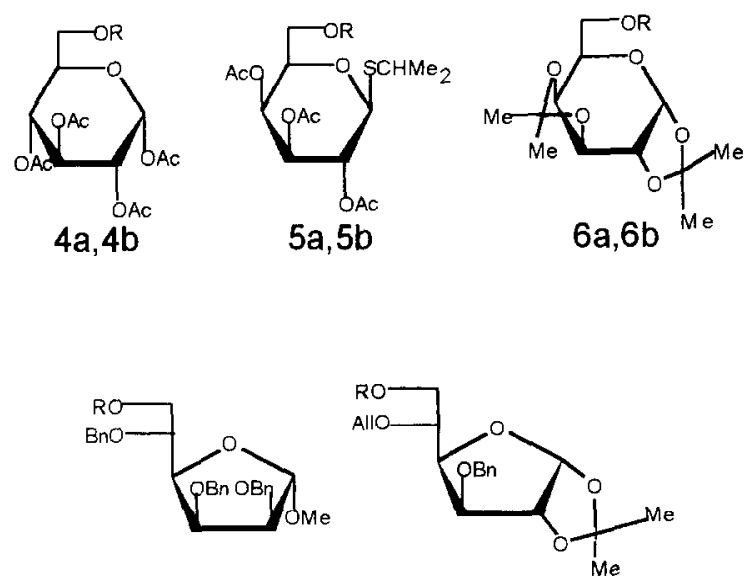

$7 a, 7 b$

$8 a, 8 b$

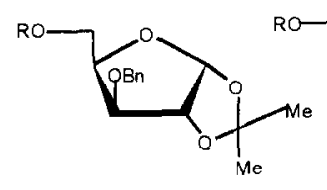

$9 a, 9 b$

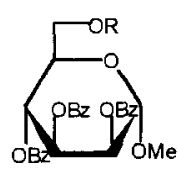

$3 a, 3 b$

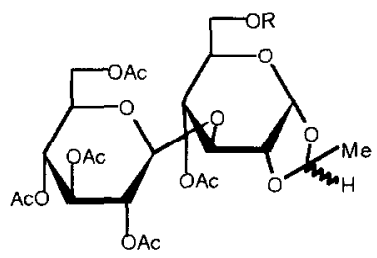

1a-12a: R = Trityl

1b-12b: $R=H$
$12 a, 12 b$

Table 1

Optical rotation data for compounds 1a-11a and $1 \mathbf{b}-\mathbf{1 1 b}$

\begin{tabular}{|c|c|c|c|c|c|}
\hline Compd. & {$[\alpha]_{D}^{20}$} & Lit. [ref.] & Compd. & {$[\alpha]_{\mathrm{D}}^{20}$} & Lit. [ref.] \\
\hline $1 \mathbf{a}$ & $+23^{\circ}\left(c 4.0, \mathrm{CHCl}_{3}\right)$ & $+20^{\circ}[3]$ & 1b & $+22.9^{\circ}\left(c 3.5, \mathrm{CHCl}_{3}\right)$ & $-{ }^{a}[3]$ \\
\hline $2 \mathbf{a}$ & $+42^{\circ}\left(c 5.0, \mathrm{CHCl}_{3}\right)$ & $-{ }^{a}[15]$ & $\mathbf{2 b}$ & $+55^{\circ}\left(c 3.0, \mathrm{CHCl}_{3}\right)$ & $+55.7^{\circ}[15]$ \\
\hline 3a & $-45^{\circ}\left(\right.$ c $\left.2.0, \mathrm{CHCl}_{3}\right)$ & - & $\mathbf{3 b}$ & $-38^{\circ}\left(\right.$ c $\left.3.0, \mathrm{CHCl}_{3}\right)$ & - \\
\hline $4 \mathbf{a}$ & $+46^{\circ}(c 1.0, \mathrm{pyr})$ & $+49^{\circ}[21]$ & 4b & $+12^{\circ}\left(\right.$ c $\left.5.0, \mathrm{CHCl}_{3}\right)$ & $+11^{\circ}[21]$ \\
\hline $\mathbf{5 a}$ & $+17.5^{\circ}\left(c 4.0, \mathrm{CHCl}_{3}\right)$ & - & $\mathbf{5 b}$ & $+10^{\circ}\left(c 4.0, \mathrm{CHCl}_{3}\right)$ & - \\
\hline $\mathbf{6 a}$ & $-64.7\left(c 8.5, \mathrm{CHCl}_{3}\right)$ & $-{ }^{\mathrm{a}}[4]$ & $\mathbf{6 b}$ & $-54^{\circ}\left(c 2.0, \mathrm{CHCl}_{3}\right)$ & $-53^{\circ}[4]$ \\
\hline $7 \mathbf{a}$ & $+20^{\circ}\left(c 7.0, \mathrm{CHCl}_{3}\right)$ & - & $7 \mathbf{b}$ & $+5^{\circ}\left(c 2.0, \mathrm{CHCl}_{3}\right)$ & - \\
\hline $\mathbf{8 a}$ & $-13^{\circ}\left(c 9.0, \mathrm{CHCl}_{3}\right)$ & $-{ }^{a}[13]$ & $\mathbf{8 b}$ & $-33.6^{\circ}\left(\right.$ c $\left.12.5, \mathrm{CHCl}_{3}\right)$ & $-{ }^{a}[13]$ \\
\hline 9a & $-32^{\circ}\left(\right.$ c $\left.5.0, \mathrm{CHCl}_{3}\right)$ & $-{ }^{\mathbf{a}}$ [4] & $9 \mathbf{b}$ & $-58.7^{\circ}\left(c 7.5, \mathrm{CHCl}_{3}\right)$ & $-{ }^{a}[4]$ \\
\hline 10a & $+56^{\circ}\left(c 5.0, \mathrm{CHCl}_{3}\right)$ & $-{ }^{a}[22]$ & 10b & $+80^{\circ}\left(c 5.0, \mathrm{CHCl}_{3}\right)$ & $-{ }^{a}[22]$ \\
\hline $11 \mathbf{a}$ & $-40^{\circ}\left(c 5.0, \mathrm{CHCl}_{3}\right)$ & $-^{\mathrm{a}}[23]$ & $11 \mathrm{~b}$ & $+20^{\circ}\left(c 1.0, \mathrm{CHCl}_{3}\right)$ & $+23^{\circ}[23]$ \\
\hline
\end{tabular}

a No optical rotation data were reported for this compound in the cited article. 
In summary, we provide a very effective and facile method for detritylation under quite mild conditions, a method that can be used in the synthesis of oligosaccharides.

\section{Experimental}

General methods. - Optical rotations were determined at $20{ }^{\circ} \mathrm{C}$ with a Perkin-Elmer Model 241-MC automatic polarimeter. ' $\mathrm{H}$ NMR spectra were recorded with Varian XL-400 and Varian XL-200 spectrometers for solns in $\mathrm{CDCl}_{3}$. Chemical shifts are given in $\mathrm{ppm}$ downfield from internal $\mathrm{Me}_{4} \mathrm{Si}$. TLC was performed on Silica Gels $\mathrm{G}$ and $\mathrm{HF}$, with detection either by charring with $30 \%(\mathrm{v} / \mathrm{v}) \mathrm{H}_{2} \mathrm{SO}_{4}$ and $\mathrm{MeOH}$ or by UV light.

Detritylation of compounds $\mathbf{1 a}-\mathbf{1 2 \mathbf { a }}$ - - To a solution of tritylated sugar $(50-100 \mathrm{mg})(\mathbf{1 a - 1 2 a})$ in $\mathrm{CH}_{2} \mathrm{Cl}_{2}(2 \mathrm{~mL})$ was added solid $\mathrm{FeCl}_{3} \cdot 6 \mathrm{H}_{2} \mathrm{O}$ (commercial product, without any pretreatment, 2 equiv). The mixture was stirred at room temperature for $1 \mathrm{~h}$, after which time the reaction was complete as indicated by TLC (2:1 petroleum ether-EtOAc). Water was added, and the mixture was diluted with $\mathrm{CH}_{2} \mathrm{Cl}_{2}$. The organic layers were combined, dried over $\mathrm{Na}_{2} \mathrm{SO}_{4}$, and concentrated to give quantitatively the desired product $(\mathbf{1} \mathbf{b}-\mathbf{1 2 b})$.

The $\mathrm{H}$ NMR data of some compounds $\left(\mathrm{CDCl}_{3}\right.$, $\mathrm{Me}_{4} \mathrm{Si}$ as internal standard) are as follows. 5a: $\delta$ $7.48-7.20(\mathrm{~m}, 15 \mathrm{H}, \mathrm{Ph}), 5.42(\mathrm{~d}, 1 \mathrm{H}, \mathrm{H}-3), 5.20-$ $5.10(\mathrm{~m}, 2 \mathrm{H}, \mathrm{H}-2,4), 4.58(\mathrm{~d}, 1 \mathrm{H}, \mathrm{H}-1), 4.10(\mathrm{~m}, 1$ H, H-5), 3.40-3.30 (m, $\left.2 \mathrm{H}, \mathrm{H}-6,6^{\prime}\right), 3.20(\mathrm{~m}, 1 \mathrm{H}$, $\mathrm{CH}), 2.16,2.08,1.98\left(3 \mathrm{~s}, 9 \mathrm{H}, \mathrm{COCH}_{3}\right), 1.54,1.22$ $\left(2 \mathrm{~s}, 6 \mathrm{H}, \mathrm{CH}_{3}\right) . \mathbf{5 b}: \delta 5.44\left(\mathrm{~d}, 1 \mathrm{H}, J_{3,4} 3.2 \mathrm{~Hz}\right.$, $\mathrm{H}-3), 5.18\left(\mathrm{~d}, \mathrm{i} \mathrm{H}, J_{2,1} 9.3 \mathrm{~Hz}, \mathrm{H}-2\right), 5.06(\mathrm{dd}, 1 \mathrm{H}$, $\left.J_{4.5} 10.0 \mathrm{~Hz}, \mathrm{H}-4\right), 4.62(\mathrm{~d}, 1 \mathrm{H}, \mathrm{H}-1), 3.96-3.70(\mathrm{~m}$, $\left.2 \mathrm{H}, \mathrm{H}-6,6^{\prime}\right) .7 \mathrm{a}: \delta 7.6-7.20(\mathrm{~m}, 30 \mathrm{H}, \mathrm{Ph}), 4.92(\mathrm{~d}$, $1 \mathrm{H}, \mathrm{H}-1), 3.50,3.22\left(2 \mathrm{~d}, 2 \mathrm{H}, \mathrm{H}-6,6^{\prime}\right) .7 \mathbf{b}: \delta$ $7.30-7.10(\mathrm{~m}, 15 \mathrm{H}, \mathrm{Ph}), 5.04\left(\mathrm{~d}, 1 \mathrm{H}, J_{1.2} 3.3 \mathrm{~Hz}\right.$, $\mathrm{H}-1), 4.86\left(\mathrm{~d}, 1 \mathrm{H}, J_{3.4} 11.4 \mathrm{~Hz}, \mathrm{H}-3\right), 4.69,4.62$ (2 d, $\left.2 \mathrm{H}, J 11.0 \mathrm{~Hz}, \mathrm{CH} \mathrm{H}_{2} \mathrm{Ph}\right), 4.59,4.49(2 \mathrm{~d}, 2 \mathrm{H}, J$ $\left.10.6 \mathrm{H} z, \mathrm{CH}_{2} \mathrm{Ph}\right), 4.48(2 \mathrm{~d}, 2 \mathrm{H}, J 11.0 \mathrm{~Hz}$, $\left.\mathrm{CH}_{2} \mathrm{Ph}\right)$, 4.24-4.22 (m, $\left.2 \mathrm{H}, \mathrm{H}-2,4\right), 3.97(\mathrm{~m}, 1 \mathrm{H}$, $\mathrm{H}-5), 3.92\left(\mathrm{dd}, \mathrm{I} \mathrm{H}, J_{5.6} 4.4, J_{6.6^{\prime}} 12.4 \mathrm{~Hz}, \mathrm{H}-6\right.$ ), $3.80\left(\mathrm{dd}, \mathrm{I} \mathrm{H}, J_{5,6^{\prime}} 2.0 \mathrm{~Hz}, \mathrm{H}-6^{\prime}\right) .8 \mathbf{a}: \delta \quad 7.58-7.10$ $(\mathrm{m}, 20 \mathrm{H} . \mathrm{Ph}), 5.81\left(\mathrm{~d}, 1 \mathrm{H}, J_{1.2} 3.7 \mathrm{~Hz}, \mathrm{H}-1\right)$, 5.89-5.72 (m, I H, CH of All), 5.20-5.00 $(\mathrm{m}, 2 \mathrm{H}$, $\mathrm{CH}_{2}$ of All $), 4.65,4.50(2 \mathrm{~d}, 2 \mathrm{H}, J 11.6 \mathrm{~Hz}$, $\left.\mathrm{OCH}_{2} \mathrm{Ph}\right), 4.55(\mathrm{~d}, 1 \mathrm{H}, \mathrm{H}-2), 4.43\left(\mathrm{dd}, 1 \mathrm{H}, J_{3.4}\right.$ $\left.2.9, J_{4,5} 9.0 \mathrm{~Hz}, \mathrm{H}-4\right), 4.10(\mathrm{~d}, 1 \mathrm{H}, \mathrm{H}-3), 4.20-4.05$ $\left(\mathrm{m}, 2 \mathrm{H}, \mathrm{CH}_{2}\right.$ of All), $3.88(\mathrm{~m}, 1 \mathrm{H}, \mathrm{H}-5), 3.48(\mathrm{dd}, 1$ $\left.\mathrm{H}, J_{5.6} 1.7, J_{5,6^{\prime}} 10.5 \mathrm{~Hz}, \mathrm{H}-6\right), 3.40\left(\mathrm{dd}, 1 \mathrm{H}, J_{5.6}\right.$ $\left.5.1 \mathrm{~Hz}, \mathrm{H}-6^{\prime}\right), 1.44,1.25\left(2 \mathrm{~s}, 6 \mathrm{H}, \mathrm{CH}_{3}\right) .8 \mathbf{b}: \delta$ $7.40-7.30(\mathrm{~m}, 5 \mathrm{H}, \mathrm{Ph}), 5.90\left(\mathrm{~d}, 1 \mathrm{H}, J_{1.2} 3.9 \mathrm{~Hz}\right.$, $\mathrm{H}-1), 4.00-3.76\left(\mathrm{~m}, 3 \mathrm{H}, \mathrm{H}-5,6,6^{\prime}\right), 3.00(\mathrm{~s}, 1 \mathrm{H}$. OH). 9a: $\delta 7.45-7.25(\mathrm{~m}, 20 \mathrm{H}, \mathrm{Ph}), 5.90(\mathrm{~d}, 1 \mathrm{H}$, $\left.J_{1.2} 3.3 \mathrm{~Hz}, \mathrm{H}-1\right), 4.58\left(\mathrm{~d}, 1 \mathrm{H}, J_{3.4} 4.7 \mathrm{~Hz}, \mathrm{H}-3\right)$, $4.59,4.42\left(2 \mathrm{~d}, 2 \mathrm{H}, J 12.7 \mathrm{~Hz}, \mathrm{CH}_{2} \mathrm{Ph}\right), 4.37(\mathrm{~m}, 1$ $\mathrm{H}, \mathrm{H}-4), 4.00(\mathrm{~d}, 1 \mathrm{H}, \mathrm{H}-2), 3.55\left(\mathrm{dd}, 1 \mathrm{H}, J_{4.5}, J_{5.5^{\prime}}\right.$ $8.9 \mathrm{~Hz}, \mathrm{H}-5), 3.30\left(\mathrm{dd}, 1 \mathrm{H}, J_{4,5^{\prime}} 6.6 \mathrm{~Hz}, \mathrm{H}-5^{\prime}\right), 1.51$, $1.30\left(2 \mathrm{~s}, 6 \mathrm{H}, \mathrm{CH}_{3}\right) .9 \mathrm{~b}: \delta 7.40-7.20(\mathrm{~m}, 5 \mathrm{H}, \mathrm{Ph})$, $5.95(\mathrm{~d}, 1 \mathrm{H}, \mathrm{H}-1), 4.00-3.80\left(\mathrm{~m}, 3 \mathrm{H}, \mathrm{H}-2.5,5^{\prime}\right)$, $1.86(\mathrm{~s}, 1 \mathrm{H}, \mathrm{OH}) .10 \mathrm{a}: \delta 7.45-7.25(\mathrm{~m}, 20 \mathrm{H}, \mathrm{Ph})$, $5.84\left(\mathrm{~d}, 1 \mathrm{H}, J_{1.2} 3.0 \mathrm{~Hz}, \mathrm{H}-1\right), 4.58\left(\mathrm{~d}, 1 \mathrm{H}, J_{2.3}\right.$ $\left.7.5, J_{3.4} 3.5 \mathrm{~Hz}, \mathrm{H}-3\right), 4.60,4.38(2 \mathrm{~d}, 2 \mathrm{H}, J 10.5$ $\left.\mathrm{Hz}, \mathrm{CH}_{2} \mathrm{Ph}\right), 4.20(\mathrm{~m}, 1 \mathrm{H}, \mathrm{H}-4), 4.00\left(\mathrm{dd}, 1 \mathrm{H}, J_{2.3}\right.$ $10.0 \mathrm{~Hz}, \mathrm{H}-2), 3.50\left(\mathrm{dd}, 1 \mathrm{H}, J_{4.5} 2.5, J_{5.5^{\prime}} 9.5 \mathrm{~Hz}\right.$, $\mathrm{H}-5), 3.30\left(\mathrm{dd}, 1 \mathrm{H}, J_{4,5}, 3.5 \mathrm{~Hz}, \mathrm{H}-5^{\prime}\right), 1.51,1.30(2$ $\left.\mathrm{s}, 6 \mathrm{H}, \mathrm{CH}_{3}\right) .10 \mathrm{~b}: \delta 7.40-7.20(\mathrm{~m}, 5 \mathrm{H}, \mathrm{Ph}), 5.90$ (d, 1 H, H-1), 3.80-3.60 (m, $\left.2 \mathrm{H}, \mathrm{H}-6,6^{\prime}\right), 3.20(\mathrm{~s}, 1$ $\mathrm{H}, \mathrm{OH}) .11 \mathrm{a}: \delta 7.30-7.10(\mathrm{~m}, 15 \mathrm{H}, \mathrm{Ph}), 5.85(\mathrm{~d}, \mathrm{I}$ $\left.\mathrm{H}, J_{\mathrm{I}, 2} 4.0 \mathrm{~Hz}, \mathrm{H}-1\right), 5.38\left(\mathrm{~d}, 1 \mathrm{H}, J_{3.4} 3.8 \mathrm{~Hz}, \mathrm{H}-3\right)$, $4.48(\mathrm{~d}, 1 \mathrm{H}, \mathrm{H}-2), 4.55-4.40(\mathrm{~m}, \mathrm{I} \mathrm{H}, \mathrm{H}-4), 3.45$ $\left(\mathrm{dd}, 1 \mathrm{H}, J_{4.5} 4.0, J_{5,5^{\prime}} 8.0 \mathrm{~Hz} . \mathrm{H}-5\right), 3.20(\mathrm{dd}, 1 \mathrm{H}$, $\left.J_{4,5^{\prime}} 8.0 \mathrm{~Hz}, \mathrm{H}^{\prime}\right), 2.00\left(\mathrm{~s}, 3 \mathrm{H}, \mathrm{COCH}_{3}\right), 1.52,1.26$ $\left(2 \mathrm{~s}, 6 \mathrm{H}, \mathrm{CH}_{3}\right) .11 \mathrm{~b}: \delta 5.93\left(\mathrm{~d} .1 \mathrm{H}, J_{1.2} 3.6 \mathrm{~Hz}\right.$, $\mathrm{H}-1), 4.00\left(\mathrm{dd}, 1 \mathrm{H}, J_{4.5} 2.4, J_{5.5}, 5.6 \mathrm{~Hz}, \mathrm{H}-5\right), 3.80$ (dd, $\left.1 \mathrm{H}, J_{4.5^{\prime}} 2.2 \mathrm{~Hz}, \mathrm{H}-5^{\prime}\right), 3.20(\mathrm{~s}, 1 \mathrm{H}, \mathrm{OH}), 2.03$ $\left(\mathrm{s}, 3 \mathrm{H}, \mathrm{COC} H_{3}\right), 1.52,1.26\left(2 \mathrm{~s}, 6 \mathrm{H}, \mathrm{CH}_{3}\right) .12 \mathrm{a}: \delta$ 7.44-7.20 (m, 15 H, Ph), 5.64 (d. 1 H, H-1), 3.30, $3.10\left(2 \mathrm{~d}, 2 \mathrm{H}, \mathrm{H}-6,6^{\prime}\right) .12 \mathrm{~b}: \delta 5.60(\mathrm{~d}, 1 \mathrm{H}, \mathrm{H}-1)$, $3.70,3.60\left(2 \mathrm{~d}, 2 \mathrm{H}, \mathrm{H}-6,6^{\prime}\right)$.

\section{Acknowledgements}

Project 29672049 was supported by The National Natural Science Foundation.

\section{References}

[1] (a) K.I. Agarwal, A. Yamazaki, P.L. Cashion, and H.G. Khorana, Angew. Chem. Int. Ed. Engl., (1972) 451-459; (b) J. Stanêk, Top. Curr. Chem., 154 ( 1990) 234-242.

[2] F. Micheel, Ber., 65 (1932) 262-265.

[3] S.J. Soudheimer, R. Eby, and C. Schuerch, Carbohydr. Res., 60 (1978) 187-192.

[4] M. Bessodes, D. Komiotis, and K. Antonakis, Tetrahedron Lett., 27 (1986) 579-580.

[5] P.E. Verkade, J. Vander Lee, and W. Meerburg, Rec. Trav. Chim., 54 (1935) 716-724.

[6] Y.M. Choy and A.M. Unrau, Carbohydr. Res., 17 (1971) 439-443.

[7] (a) N. Roy and T.E. Timell, Carbohydr. Res., 7 
(1968) 82-83; (b) G.R. Barker, Methods Carbohydr. Chem., 2 (1963) 168-172.

[8] B. Helferich, Adv. Carbohydr. Chem., 3 (1948) $79-$ 111.

[9] C.W. Baker and R.L. Whistler, Carbohydr. Res., 33 (1974) 372-374.

[10] M. Maccoss and D.J. Cameron, Carbohydr. Res., 60 (1978) 206-209.

[11] G. Randazzo, R. Capasso, M.R. Cicala, and A. Evidente, Carbohydr. Res., 85 (1980) 298-301.

[12] V. Kohli, H. Blöcker, and H. Köster, Tetrahedron Lett., 21 (1980) 2683-2686.

[13] K. Dax, W. Wolflehner, and H. Weidmann, Carbohydr. Res., 65 (1978) 132-138.

[14] R. Albert, K. Dax, R. Pleschko, and A.E. Stütz, Carbohydr. Res., 37 (1985) 282-290.

[15] A. Klemer, M. Bieber, and H. Wiburs, Liebigs Ann. Chem., (1983) 1416-1421.
[16] P. Kovăc and C.P.I. Glaudemans, Carbohydr. Res., 140 (1985) 313-318.

[17] H. Köster and N.D. Sinha, Tetrahedron Lett., 23 (1982) 2641-2464.

[18] N.K. Kochetkov, B.A. Dmitriev, N.E. Bairamova, and A.V. Nikolaev, Izv. Akad. Nauk SSSR, Ser. Khim., (1978) 652-656; Chem. Abstr., 89 (1978) $24655 f$.

[19] R. Rodebaugh, J.S. Debenham, and B. Fraser-Reid, Tetrahedron Lett., 37 (1996) 5477-5478.

[20] N. Ikemoto, O.K. Kim, L.C. Lo, V. Satyanarayana, M. Chang, and K. Nakanishi, Tetrahedron Lett., 33 (1992) 4295-4298.

[21] M.A. Mohanel, Abd El-Rahman, and U. Hovnemain, Carbohydr. Res., 38 (1974) 355-358.

[22] W. Sowa, Can. J. Chem., 46 (1968) 1586-1589.

[23] Y. Ishido, N. Sabuiri, M. Sekiga, and N. Nakazaki, Carbohydr. Res., 97 (1981) 51-79. 